\title{
Theory of a Practical Delaunay Meshing Algorithm for a Large Class of Domains *
}

\author{
Siu-Wing Cheng ${ }^{\dagger} \quad$ Tamal K. Dey ${ }^{\ddagger} \quad$ Joshua Levine $^{\S}$
}

\begin{abstract}
Recently a Delaunay refinement algorithm has been proposed that can mesh domains as general as piecewise smooth complexes. These domains include polyhedra, smooth and piecewise smooth surfaces, volumes enclosed by them, and above all non-manifold spaces. The algorithm is guaranteed to capture the input topology at the expense of four tests, some of which are computationally intensive and hard to implement. The goal of this paper is to present the theory that justifies a refinement algorithm with a single disk test in place of four tests of the previous algorithm.

The algorithm is supplied with a resolution parameter that controls the level of refinement. We prove that, when the resolution is fine enough (this level is reached very fast in practice), the output mesh becomes homeomorphic to the input while preserving all input features. Moreover, regardless of the refinement level, each $k$-manifold element in the input complex is meshed with a triangulated $k$-manifold. Boundary incidences among elements maintain the input structure. Implementation results reported in a companion paper corroborate our claims.
\end{abstract}

\footnotetext{
*Research supported by NSF, USA (CCF-0430735 and CCF-0635008) and RGC, Hong Kong, China (HKUST 6181/04E).

${ }^{\dagger}$ Department of Computer Science and Engineering, HKUST, Hong Kong. Email: scheng@cse.ust.hk

${ }^{\ddagger}$ Department of Computer Science and Engineering, Ohio State University, Ohio, USA. Email: tamaldey@cse.ohio-state.edu

$\S$ Department of Computer Science and Engineering, Ohio State University, Ohio, USA. Email: levinej@cse.ohio-state.edu
} 


\section{Introduction}

Delaunay meshing of geometric domains is sought in a number of applications in science and engineering. Since its introduction by Chew [11], the Delaunay refinement technique has been growing in its application to meshing geometric domains. Starting with polygonal and polyhedral complexes $[13,17,18,21,22]$ where quality of elements were of prime concern, the technique has been extended to smooth domains where topology preservation is a major issue $[4,5,10,12,19]$. Nonsmooth curved domains, the next in the order of difficulty, pose some fundamental obstacles to Delaunay refinement on which research results have started to appear.

There are two main challenges faced in extending Delaunay refinement to non-smooth domains. First, the sampling theory developed for smooth surfaces [1] is not applicable to non-smooth surfaces. Secondly, as in the polyhedral case $[9,7,20,23]$, small input angles possibly present at non-smooth regions pose problems for the termination of Delaunay refinement. Boissonnat and Oudot [3] successfully extended their algorithm for smooth surfaces to non-smooth ones but failed to admit small input angles. Cheng, Dey, and Ramos [8] removed this constraint on input angles enabling their algorithm to work on a large class of domains called piecewise smooth complex (PSC). This class includes polyhedral domains, smooth and piecewise smooth surfaces, volumes enclosed by them, and even non-manifolds. This algorithm protects non-smooth curves and vertices in the input complex with balls that are turned into weighted points during refinement stage. Staying away from non-smooth regions, the algorithm can afford to admit arbitrary small input angles. Notwithstanding its theoretical success, practical validity of the algorithm remains questionable since it employs costly computations during iterative refinement. The goal of this paper is to develop the theory further so that a simpler refinement strategy can be devised making it viable in practice.

The refinement procedure of Cheng et al. [8] performs four tests to guarantee topology preservation, namely (i) a Voronoi edge does not intersect the domain more than once, (ii) normals on the curves and surface patches do not vary beyond a threshold within Voronoi cells, (iii) no Delaunay edge in the restricted triangulation (defined later) connect vertices across different patches, and (iv) the restricted Delaunay triangles incident to points in a patch make a topological disk. After collecting restricted triangles, that is, triangles dual to the Voronoi edges intersecting the input domain, tests (i) and (iv) are only combinatorial. However, test (ii) is quite expensive and is a major obstacle in making the algorithm practical. Also, test (iii) requires recognizing intersections of Voronoi facets with the domain which is computationally harder than recognizing Voronoi edge-domain intersections.

We replace four tests with a single test that checks a topological disk condition similar to (iv). As long as the restricted triangles incident to each point do not form a topological disk, the refinement routine samples a new point from the domain. We argue that this procedure terminates. We prove the following guarantees. The algorithm is supplied with an input resolution parameter that determines how refined the output mesh should be. We prove that, regardless of the refinement level, a $k$-manifold in the input is meshed with a simplicial $k$-manifold. Boundary incidences among different manifold elements in the input complex are maintained in the output. Furthermore, if the refinement is sufficiently dense, homeomorphism between input and output is reached. More importantly, this refinement level is achieved very fast in practice giving us a provable practical algorithm for Delaunay meshing for a

vast array of domains. We report the practical results in detail in a companion paper [6] (a sample is in Figure 1).

\section{Preliminaries}

2.1 Domain Throughout this paper, we assume a generic intersection property that a $k$-manifold $\sigma \subset \mathbb{R}^{3}, 0 \leqslant k \leqslant 3$, and a $j$-manifold $\sigma^{\prime} \subset \mathbb{R}^{3}, 0 \leqslant j \leqslant 3$, intersect (if at all) in a $(k+j-3$ )-manifold 

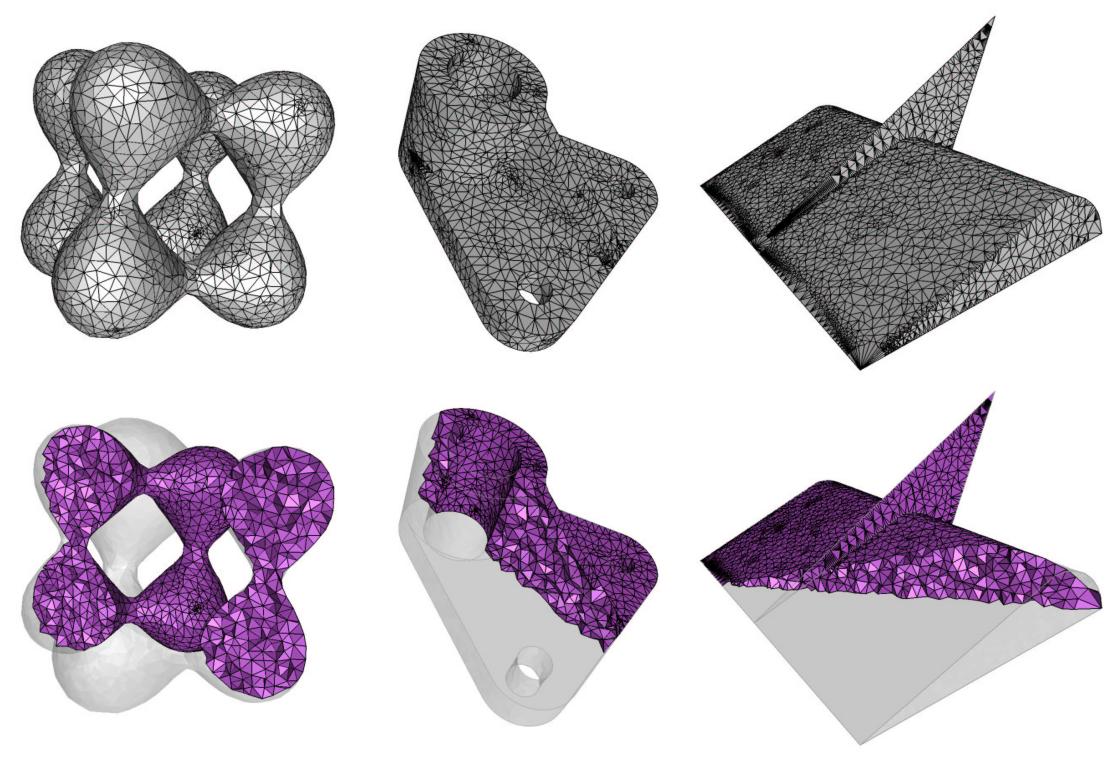

Figure 1: Meshed PSCs, Metaball (Smooth), Part (Manifold PSC), and Wedge (Non-manifold, PSC with small angles). Top row: surface mesh, bottom row: volume mesh.

if $\sigma \not \subset \sigma^{\prime}$ and $\sigma^{\prime} \not \subset \sigma$. We will use both geometric and topological versions of closed balls. A geometric closed ball centered at point $x \in \mathbb{R}^{3}$ with radius $r>0$, is denoted as $B(x, r)$. We use int $\mathbb{X}$ and bd $\mathbb{X}$ to denote the interior and boundary of a topological space $\mathbb{X}$, respectively.

The domain $\mathcal{D}$ is a piecewise smooth complex (PSC) where each element is a compact subset of a smooth $\left(C^{2}\right) k$-manifold, $0 \leqslant k \leqslant 3$. Each element is closed and hence contains its boundaries (possibly empty). We use $\mathcal{D}_{k}$ to denote the subset of all $k$-dimensional elements, the $k$ th stratum. $\mathcal{D}_{0}$ is a set of vertices; $\mathcal{D}_{1}$ is a set of curves called 1-faces; $\mathcal{D}_{2}$ is a set of surface patches called 2-faces; $\mathcal{D}_{3}$ is a set of volumes called 3-faces. For $1 \leqslant k \leqslant 2$, we use $\mathcal{D}_{\leqslant k}$ to denote $\mathcal{D}_{0} \cup \ldots \cup \mathcal{D}_{k}$.

The domain $\mathcal{D}$ satisfies the usual proper requirements for being a complex: (i) interiors of the elements are pairwise disjoint and for any $\sigma \in \mathcal{D}$, bd $\sigma \subset \mathcal{D}$; (ii) for any $\sigma, \sigma^{\prime} \in \mathcal{D}$, either $\sigma \cap \sigma^{\prime}=\emptyset$ or $\sigma \cap \sigma^{\prime}$ is a union of cells in $\mathcal{D}$. We use $|\mathcal{D}|$ to denote the underlying space of $\mathcal{D}$. For $0 \leqslant k \leqslant 3$, we also use $\left|\mathcal{D}_{k}\right|$ to denote the underlying space of $\mathcal{D}_{k}$.

For any point $x$ on a 2 -face $\sigma$, we use $n_{\sigma}(x)$ to denote a unit outward normal to the surface of $\sigma$ at $x$. For any point $x$ on a 1 -face $\sigma, n_{\sigma}(x)$ denotes a unit oriented tangent to the curve of $\sigma$ at $x$. (We assume a consistent orientation of the tangents.)

2.2 Complexes We will be dealing with weighted points and their Delaunay and Voronoi diagram. A weighted point $p$ is represented as a ball $\hat{p}=B\left(p, w_{p}\right)$. The squared weighted distance of any point $x \in \mathbb{R}^{3}$ from $\hat{p}$ is given by $\|x-p\|^{2}-w_{p}^{2}$. Under this distance metric, one can define weighted versions of Delaunay and Voronoi diagram. For a weighted point set $S \subset \mathbb{R}^{3}$, let Vor $S$ and Del $S$ denote the weighted Voronoi and Delaunay diagrams of $S$ respectively. Each diagram is a cell complex where each $k$-face is a $k$-polytope in Vor $S$ and is a $k$-simplex in Del $S$. Each $k$-simplex $\xi$ in Del $S$ is dual to a $(3-k)$-face $V_{\xi}$ in Vor $S$ and vice versa.

Let $S$ be a point set sampled from $|\mathcal{D}|$. For any sub-collection $\mathbb{X} \subset \mathcal{D}$ we define $\left.\operatorname{Del} S\right|_{\mathbb{X}}$ to be the Delaunay subcomplex restricted to $\mathbb{X}$, i.e., each simplex $\left.\xi \in \operatorname{Del} S\right|_{\mathbb{X}}$, called a restricted simplex, is the dual of a Voronoi face $V_{\xi}$ where $\left.V_{\xi}\right|_{\mathbb{X}}=V_{\xi} \cap|\mathbb{X}| \neq \emptyset$. By this definition, for any $\sigma \in \mathcal{D}$, Del $\left.S\right|_{\sigma}$ denote 
the Delaunay subcomplex restricted to $\sigma$ and

$$
\left.\operatorname{Del} S\right|_{\mathcal{D}_{i}}=\left.\bigcup_{\sigma \in \mathcal{D}_{i}} \operatorname{Del} S\right|_{\sigma},\left.\quad \operatorname{Del} S\right|_{\mathcal{D}}=\left.\bigcup_{\sigma \in \mathcal{D}} \operatorname{Del} S\right|_{\sigma}
$$

An $i$-face $\sigma \in \mathcal{D}_{i}$ should be meshed with $i$-simplices. However, Del $\left.S\right|_{\sigma}$ may have lower dimensional simplices not incident to any restricted $i$-simplex. Therefore, we compute special sub-complexes of restricted complexes. For $\sigma \in \mathcal{D}_{i}$, let $\left.\mathrm{Skl}^{i} S\right|_{\sigma}$ denote the following $i$-dimensional subcomplex of Del $\left.S\right|_{\sigma}$ :

$$
\left.\mathrm{Skl}^{i} S\right|_{\sigma}=\text { closure }\left\{t|t \in \operatorname{Del} S|_{\sigma} \text { is an } i \text {-simplex }\right\} \text {. }
$$

We extend the definition to strata:

$$
\left.\mathrm{Skl}^{i} S\right|_{\mathcal{D}_{i}}=\left.\bigcup_{\sigma \in \mathcal{D}_{i}} \mathrm{Skl}^{i} S\right|_{\sigma}
$$

2.3 Refinement Our strategy is to run Delaunay refinement with only the disk condition formulated as follows. See Figure 2 for more explanations.

Let $p$ be a point on a 2-face $\sigma$. Let $\operatorname{Umb}_{\mathcal{D}}(p)$ and $\operatorname{Umb}_{\sigma}(p)$ be the set of triangles in $\left.\mathrm{Skl}^{2} S\right|_{\mathcal{D}_{2}}$ and $\left.\mathrm{Skl}^{2} S\right|_{\sigma}$ respectively which are incident to $p$. The following disk condition is used for refinement. Once the restricted Delaunay triangles are collected, this check is only combinatorial.

Disk_Condition $(p)$ : (i) $\operatorname{Umb}_{\mathcal{D}}(p)=\bigcup_{\sigma \ni p} \operatorname{Umb}_{\sigma}(p)$, (ii) for each $\sigma \in \mathcal{D}_{2}$ containing $p$, underlying space of $\operatorname{Umb}_{\sigma}(p)$ is a 2-disk which has all vertices in $\sigma$. Point $p$ is in the interior of this 2-disk if and only if $p \in \operatorname{int} \sigma$. Also, if $p$ is in $\operatorname{bd} \sigma$, it is not connected to any other point on $\mathcal{D}_{1}$ which is not adjacent to it.
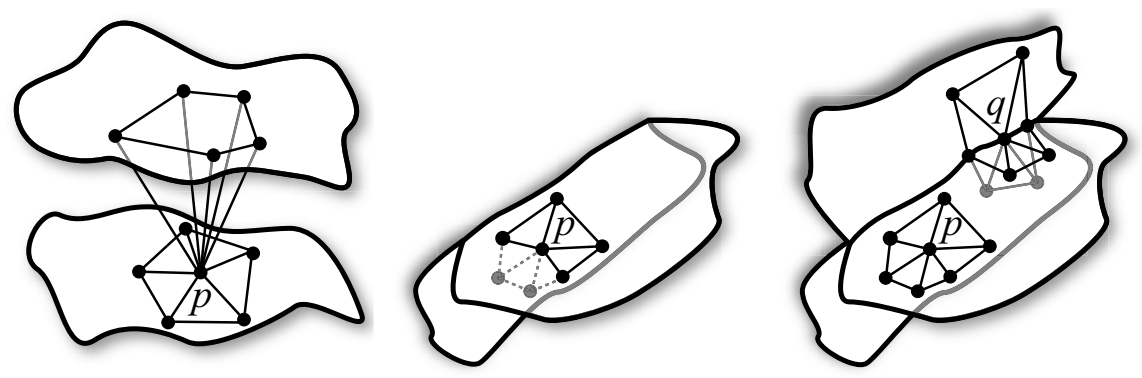

Figure 2: Disk condition: (left):point $p \in \sigma$ has a disk in $\sigma$ and another disk in $\tau \neq \sigma$ violating condition (i) (middle): point $p \in \sigma$ has a topological disk but some of its vertices (lightly shaded) belong to $\tau$ violating condition (ii), (right): Points $p$ and $q$ satisfy disk condition. Point $p$, an interior point in $\sigma$, lies in the interior of its disk in $\sigma$. Point $q$, a boundary point, has three disks for each of the three 2-faces.

\section{Protection}

The neighborhoods of the curves and vertices in $\mathcal{D}_{\leqslant 1}$ are regions of potential problems for Delaunay refinements. First, if the elements incident to these curves and vertices make small angles at the points of incidence, usual Delaunay refinement may not terminate. Second, these curves and vertices represent 'features' in the input which should be preserved in the output for many applications. Usual Delaunay refinement may destroy these features $[3,14]$. To overcome these problems Cheng et al. [8] protect elements in $\mathcal{D}_{\leqslant 1}$ with balls. We will not repeat the algorithm for computing these protecting balls here but mention only some of the key properties these balls satisfy. 
Protection properties: Let $\omega \leqslant 0.076$ be a positive constant and $\mathcal{B}_{p}$ denote the protecting ball of a point $p$.

1. Any two adjacent balls on a 1-face overlap significantly without containing each other's centers.

2. No three balls have a common intersection.

3. Let $p \in \sigma$ be the center of a protecting ball. Further, let $B=B(p, R)$ be a ball with radius $R$ and center $p$ where $R \leqslant c \operatorname{radius}\left(\mathcal{B}_{p}\right)$ for some $c \leqslant 8$.

(a) For $\tau=\sigma$ or any 2-face incident to $\sigma, \angle n_{\tau}(p), n_{\tau}(z) \leqslant 2 \omega$ for any $z \in B \cap \tau$.

(b) $B$ intersects $\sigma$ in a single open curve and any 2-face incident to $\sigma$ in a topological disk.

In practice, balls satisfying 1 and 2 can be computed easily. If they are chosen small enough 3 is satisfied as well. After computing the protecting balls, each of them is turned into a weighted vertex. That is, for each protecting ball $\mathcal{B}_{p}$, we obtain the weighted point $\left(p, w_{p}\right)$, where $w_{p}=\operatorname{radius}\left(\mathcal{B}_{p}\right)$. For technical reasons that will be clear later we need to ensure that each 2 -face is intersected by some Voronoi edge in the Voronoi diagram Vor $S$ of the current point set. The weighted vertices ensure it for 2-faces that have boundaries. For 2-faces without boundary, initially we place three weighted points satisfying the protection properties.

After protection the meshing algorithm inserts points for further Delaunay refinement. These points are not weighted. Also, the refinement step never attempts to insert a point in the interior of any of the protecting balls. This is because no Voronoi point (equidistant from three or more points) can lie inside any protecting ball since no three of them have a common intersection. In essence, our algorithm maintains a point set $S$ with the following two properties : (i) $S$ contains all weighted points placed during protection phase, and (ii) other points in $S$ are unweighted and they lie outside the protecting balls. We call such a point set admissible. The following Lemma proved in [8] is an important consequence of the protection properties.

LEMma 3.1. Let $S$ be an admissible point set. Let $p$ and $q$ be adjacent weighted vertices on a 1-face $\sigma$. Let $\sigma_{p q}$ denote the curve segment between $p$ and $q . V_{p q}$ is the only Voronoi facet in Vor $S$ that intersects $\sigma_{p q}$, and $V_{p q}$ intersects $\sigma_{p q}$ exactly once.

\section{Algorithm}

During refinement the size of the restricted triangles determines the location of the point to be inserted. For any triangle $\left.t \in \mathrm{Skl}^{2} S\right|_{\sigma}$, define $\operatorname{size}(t, \sigma)$ to be the maximum weighted distance between the vertices of $t$ and points in $\left.V_{t}\right|_{\sigma}$. Notice that if all vertices of $t$ are unweighted, the maximum weighted distance is just the maximum Euclidean distance.

When we mesh volumes, we use the standard technique of inserting circumcenters of tetrahedra that have radius-edge ratio (denoted $\rho())$ greater than a threshold, $\rho_{0} \geqslant 1$. If the insertion of the circumcenter threatens to delete any triangle in $\left.\mathrm{Skl}^{2} S\right|_{\mathcal{D}_{2}}$, the circumcenter is not inserted. In this case we say that the triangle is encroached by the circumcenter. Essentially, this strategy allows refining most of the tetrahedra except the ones near boundary. The following pseudo-code summarizes our algorithm.

DeIPSC $\left(\mathcal{D}, \lambda, \rho_{0}\right)$

1. Protection. Protect elements in $\mathcal{D}_{\leqslant 1}$ with weighted points. Insert three weighted points in each element of $\mathcal{D}_{2}$ that has no boundary. Let $S$ be the current admissible point set.

2. Mesh2Complex. 
(a) Let $(p, \sigma)$ be any tuple where $\left.p \in \operatorname{Skl}^{2} S\right|_{\sigma}$. If $\operatorname{Disk}$ _Condition $(p)$ is violated, find the triangle $t \in \operatorname{Umb}_{\mathcal{D}}(p)$ that maximizes size $(t, \sigma)$ over all $\sigma$ containing $p$ and insert $\left.x \in V_{t}\right|_{\sigma}$ that realizes $\operatorname{size}(t, \sigma)$ into $S$. Go to step $2(\mathrm{c})$.

(b) If size $(t, \sigma)>\lambda$ for some tuple $(t, \sigma)$, where $\left.t \in \mathrm{Skl}^{2} S\right|_{\sigma}$, insert $\left.x \in V_{t}\right|_{\mathcal{D}}$ that realizes size $(t, \sigma)$ into $S$.

(c) Update Del $S$ and Vor $S$.

(d) If $S$ has grown in the last execution of step 2, repeat step 2.

3. Mesh3Complex. For any tuple $(t, \sigma)$ where $t$ is a tetrahedron in $\left.\mathrm{Skl}^{3} S\right|_{\sigma}$

(a) If $\rho(t)>\rho_{0}$ insert the orthocenter of the Delaunay ball (orthoball) of $t$ into $S$ if it does not encroach any triangle in $\left.\mathrm{Skl}^{2} S\right|_{\mathcal{D}}$ or any ball $B(p, 2 r)$ where $B(p, r)$ is a protecting ball.

(b) Update Del $S$ and Vor $S$.

(c) If $S$ has grown in the last execution of step 3, repeat step 3.

4. Return $\left.\bigcup_{i} \mathrm{Skl}^{i} S\right|_{\mathcal{D}}$.

4.1 Guarantees The analysis of the algorithm establishes two main facts: (i) the algorithm terminates, (ii) at termination the output mesh satisfies properties T1-T3:

(T1) For each $\sigma \in \mathcal{D}_{1},\left.\mathrm{Skl}^{1} S\right|_{\sigma}$ is homeomorphic to $\sigma$ and two vertices are joined by an edge in Skl $\left.{ }^{1} S\right|_{\sigma}$ if and only if these two vertices are adjacent on $\sigma$.

(T2) For $0 \leqslant i \leqslant 2$ and $\sigma \in \mathcal{D}_{i},\left.\mathrm{Skl}^{i} S\right|_{\sigma}$ is a $i$-manifold with vertices only in $\sigma$. Further, $\left.\operatorname{bd~Skl}^{i} S\right|_{\sigma}=\left.\mathrm{Skl}^{i-1} S\right|_{\mathrm{bd} \sigma}$. For $i=3$, the statement is true only if the set $\left.\mathrm{Skl}^{i} S\right|_{\sigma}$ is not empty at the end of Mesh2Complex.

(T3) There exists a $\lambda>0$ so that the output mesh of $\operatorname{DeIPSC}\left(\mathcal{D}, \lambda, \rho_{0}\right)$ is homeomorphic to $\mathcal{D}$. Further, this homeomorphism respects stratification with vertex restrictions, that is, for $0 \leqslant i \leqslant 3,\left.\mathrm{Skl}^{i} S\right|_{\sigma}$ is homeomorphic to $\sigma \in \mathcal{D}_{i}$ where $\left.\mathrm{bd} \mathrm{Skl}^{i} S\right|_{\sigma}=\left.\mathrm{Skl}^{i-1} S\right|_{\mathrm{bd} \sigma}$ and vertices of $\left.\mathrm{Skl}^{i} S\right|_{\sigma}$ lie in $\sigma$.

\section{Termination}

We prove that during refinement DeIPSC maintains a positive distance between each inserted point and all other existing points. Then the compactness of $\mathcal{D}$ allows the standard packing argument to claim termination. We need some results from sampling theory $[1,2,5]$. Let $\Sigma \subset \mathbb{R}^{3}$ be a smooth $\left(C^{2}\right.$-smooth $)$ closed surface. The local feature size $f(x)$ at a point $x \in \Sigma$ is its distance to the medial axis of $\Sigma$.

Lemma 5.1. ([1, 2, 5]) Let $\varepsilon \in(0,1 / 3)$ be some constant.

(i) For any two points $x$ and $y$ in $\Sigma$ such that $\|x-y\| \leqslant \varepsilon f(x)$,

(a) the angle between the surface normals at $x$ and $y$ is at most $\varepsilon /(1-3 \varepsilon)$;

(b) the angle between $x y$ and the surface normal at $x$ is at least $\arccos (\varepsilon / 2)$.

(ii) Let pqr be a triangle with vertices on $\Sigma$ and circumradius no more than $\varepsilon f(p)$. The angle between the normal of pqr and the surface normal at $p$ is less than $7 \varepsilon$.

We use Lemma 5.1 to prove the next result. This result says that if restricted triangles incident to a point in a 2-face $\sigma$ have small sizes, $V_{p}$ intersects $\sigma$ nicely. Figure 3 explains more about its implications. 
Lemma 5.2. Let $p \in S$ be a point on a 2-face $\sigma$. Let $\sigma_{p}$ be the connected component in $\left.V_{p}\right|_{\sigma}$ containing $p$. There exists a constant $\lambda>0$ so that following holds:

If some edge of $V_{p}$ intersects $\sigma$ and $\operatorname{size}(t, \sigma)<\lambda$ for each triangle $\left.t \in \mathrm{Skl}^{2} S\right|_{\sigma}$ incident to $p$, then

(i) there is no 2-face $\tau$ where $p \notin \tau$ and $\tau$ intersects a Voronoi edge of $V_{p}$;

(ii) $\sigma_{p}=V_{p} \cap B \cap \sigma$ where $B=B(p, 2 \lambda)$ if $p$ is unweighted and $B=B\left(p, 2 \operatorname{radius}\left(\mathcal{B}_{p}\right)+2 \lambda\right)$ otherwise;

(iii) $\sigma_{p}$ is a 2-disk;

(iv) any edge of $V_{p}$ intersects $\sigma_{p}$ at most once;

(v) any facet of $V_{p}$ intersects $\sigma_{p}$ in an empty set or an open curve.

Proof. Observe that, because of protection, $p$ has a positive minimum distance to any 2-face $\tau$ not containing it. Therefore, any Voronoi edge of $V_{p}$ intersecting $\tau$ violates the size condition when $\lambda$ is sufficiently small. This proves (i).
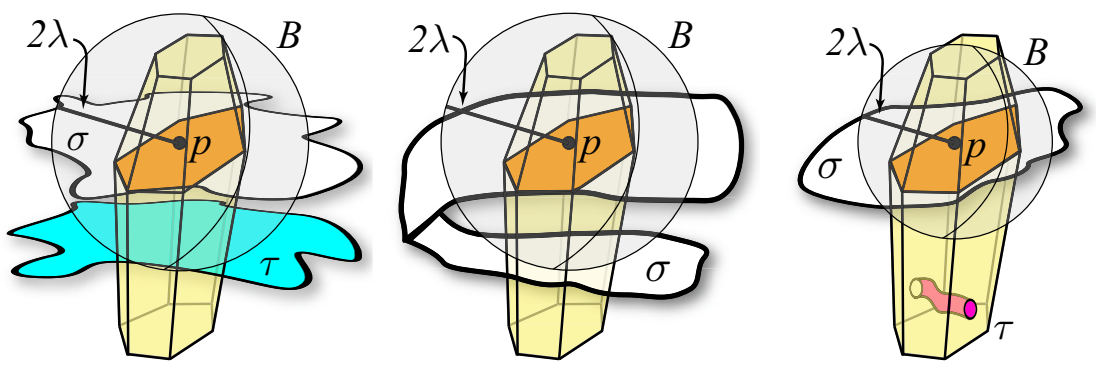

Figure 3: (left): A 2-face $\tau$ where $p \notin \tau$ intersects some edge of $V_{p}$. This is not possible according to Lemma 5.2(i), (middle): also not possible since there is another component of $\sigma$ within $B \cap V_{p}$ other than $\sigma_{p}$, (right): Within $B, \sigma$ intersects $V_{p}$ in a topological disk. It is possible that there is a different component $(\tau)$ which does not intersect any Voronoi edge and hence does not contribute any dual restricted triangle incident to $p$.

Consider $p$ is unweighted. For any facet $F$ of $V_{p}$, we use $H_{F}$ to denote the plane of $F$. Assume that $\lambda$ is less than $1 / 32$ the local feature size of the surface of $\sigma$ at $p$. Let $B=B(p, 2 \lambda)$. It is known that $B \cap \sigma$ is a 2 -disk.

First, we claim that for any facet $F$ of $V_{p}$, if $H_{F}$ intersects $B \cap \sigma$, then $\angle n_{\sigma}(p), H_{F} \leqslant \arcsin (1 / 16)$ and both $H_{F} \cap B \cap \sigma$ and $F \cap B \cap \sigma$ contains no closed curve. The dual Delaunay edge $p q$ of $F$ has length at most $4 \lambda$, which is less than $1 / 8$ the local feature size. By Lemma 5.1(ib), $\angle n_{\sigma}(p), H_{F}=\pi / 2-\angle n_{\sigma}(p), p q \leqslant \arcsin (1 / 16)$. There is no closed curve in $H_{F} \cap B \cap \sigma$ because such a closed curve would bound a 2 -disk in $B \cap \sigma$, which would contain a point $x$ such that $\angle n_{\sigma}(x), H_{F}=\pi / 2$. This is a contradiction because $\angle n_{\sigma}(x), H_{F} \leqslant \angle n_{\sigma}(x), n_{\sigma}(p)+\angle n_{\sigma}(p), H_{F} \leqslant 1 / 13+\arcsin (1 / 16)<\pi / 2$ by Lemma 5.1(ia). Since $H_{F} \cap B \cap \sigma$ contains no closed curve, neither does $F \cap B \cap \sigma$. This proves the claim.

Second, we claim that for any facet $F$ of $V_{p}$, if $H_{F}$ is within a distance of $\lambda$ from $p, H_{F} \cap B \cap \sigma$ is a single open curve. Consider the disk $H_{F} \cap B$. Let $\vec{d}$ be the projection of $n_{\sigma}(p)$ onto $H_{F}$. Let $L \subset H_{F}$ be the line through the center of $H_{F} \cap B$ orthogonal to $\vec{d}$. Let $x$ be any point in $H_{F} \cap B \cap \sigma$. The angle between $p x$ and the tangent plane at $p$ is at most $\arcsin (1 / 32)$ by Lemma $5.1(\mathrm{ib})$. We already proved that $\angle n_{\sigma}(p), H_{F} \leqslant \arcsin (1 / 16)$. So the distance between $x$ and $L$ is less than 
$\|p-x\| \sin (2 \arcsin (1 / 16)) \leqslant 2 \lambda \sin (2 \arcsin (1 / 16))<0.25 \lambda$. Let $L^{*} \subset H_{F}$ be the strip of points at distance $0.25 \lambda$ or less from $L$. Since radius of $B$ is $2 \lambda$ and $H_{F}$ is at most $\lambda$ distance from $p$, the radius of $H_{F} \cap B$ is at least $\sqrt{3} \lambda$. It follows that the boundary of $H_{F} \cap B$ intersects $L^{*}$ in two disjoint circular arcs. We already proved that there is no closed curve in $H_{F} \cap B \cap \sigma$. It can be shown that if $H_{F} \cap B \cap \sigma$ contains two open curves, one of the curves, say $C$, must have both endpoints on the same arc in $H_{F} \cap B \cap L^{*}$. The radius of $H_{F} \cap B$ is at least $\sqrt{3} \lambda$. So some tangent to $C$ must make an angle at most $\arcsin (0.25 / \sqrt{3})<0.15$ with $\vec{d}$. But this implies that the angle between the surface normal at some point on $C$ and $n_{\sigma}(p)$ is at least $\pi / 2-0.15-\angle n_{\sigma}(p), H_{F} \geqslant \pi / 2-0.15-\arcsin (1 / 16)>1$, contradicting Lemma 5.1(ia). This proves the claim.

Third, we claim that for any facet $F$ of $V_{p}$, if $F$ intersects $B \cap \sigma, F \cap B \cap \sigma$ is a single open curve with endpoints in bd $F$. We already proved that there is no closed curve in $F \cap B \cap \sigma$. Since $F$ does not have any tangential contact with $\sigma, F \cap B \cap \sigma$ is a set of open curves and the endpoints of any open curve in $F \cap \sigma$ thus lie in bd $F$. Assume to the contrary that $F \cap B \cap \sigma$ contains two open curves, say $\xi$ and $\xi^{\prime}$. By our assumption, $H_{F}$ is within a distance of $\lambda$ from $p$. We have shown before that $H_{F} \cap B \cap \sigma$ is a single open curve. Follow $H_{F} \cap B \cap \sigma$ from $\xi$ to $\xi^{\prime}$. When we leave $\xi$, we must leave $F$ at a Voronoi edge $e \subset \operatorname{bd} F$. Afterwards, we stay in the plane $H_{F}$ and we must cross the support line of $e$ again in order to reach $\xi^{\prime}$. Therefore, some tangent to $H_{F} \cap B \cap \sigma$ is parallel to $e$. However, the angle between the surface normal at some point on $H_{F} \cap B \cap \sigma$ and $n_{\sigma}(p)$ would then be at least $\pi / 2-\angle n_{\sigma}(p), e \geqslant \pi / 2-7 / 32$ by Lemma 5.1(ii). This contradicts Lemma 5.1(ia). This proves the claim.

Each facet of $V_{p}$ that intersects $B \cap \sigma$ intersects it in a single open curve. Every curve endpoint is dual to some triangle incident to $p$. Thus, our assumption about sizes of restricted triangles incident to $p$ ensures that every curve endpoint lies strictly inside $B$. This implies that that the facets of $V_{p}$ intersect $B \cap \sigma$ in a set of simple closed curves. We have analyzed this situation in [10] and showed that exactly one face in this arrangement of closed curves lies inside $V_{p}$ and it is a 2-disk. Of course, this face is $\sigma_{p}$. This proves that $\sigma_{p}=V_{p} \cap B \cap \sigma$ and it is a 2-disk. Take an edge $e$ of $V_{p}$ that intersects $B \cap \sigma$. By Lemma 5.1(ii), $\angle n_{\sigma}(p), e \leqslant 7 / 32$. Then, by Lemma 5.1(ia), $B \cap \sigma$ is monotone in the direction of $e$. Therefore, $e$ intersects $B \cap \sigma$ exactly once. It follows that any edge of $V_{p}$ intersects $\sigma_{p}$ at most once. The correctness of (iii) follows from the third claim.

The above proves the lemma for the case that $p$ is unweighted. The case of $p$ being weighted can be handled similarly by setting $B$ to be $B\left(p, 2 \operatorname{radius}\left(\mathcal{B}_{p}\right)+2 \lambda\right)$.

Now, we are ready to prove the termination of DelPSC.

\section{TheOREM 5.1. DelPSC terminates.}

Proof. Consider a vertex $p$ on a 2-face $\sigma$. Let $\lambda$ be a constant that satisfies Lemma 5.2. By Lemma 5.2(i), no restricted triangle incident to $p$ connects it to a vertex in $\tau$ where $p \notin \tau$. Notice that, because of protection, we can assume $\lambda$ to be so small that no triangle incident to $p$ connects two non-adjacent weighted vertices. This satisfies parts of disk condition. The only thing we need to show is that the restricted triangles incident to $p$ form a topological disk if $\operatorname{size}(t, \sigma)<\lambda$ for each such triangle $t$.

Observe that if no edge of the Voronoi cell $V_{p}$ intersects $\sigma$, there is no restricted triangle incident to $p$ and hence $p$ does not exist in $\left.\mathrm{Skl}^{2} S\right|_{\mathcal{D}_{2}}$ and can be ignored. So, assume that some edge of $V_{p}$ intersects $\sigma$. Let $\sigma_{p}$ be the connected component of $\left.V_{p}\right|_{\sigma}$ containing $p$. By duality the conclusions in Lemma 5.2(ii)-(v) imply that the restricted triangles incident to $p$ form a topological disk which contains $p$ in the interior if and only if $p$ lies in the interior of $\sigma$. In other words, disk condition is satisfied. Therefore, if disk condition is not satisfied for some point $p$, we can assume there is a triangle $t$ incident to $p$ for which size $(t, \sigma)>\lambda>0$. In that case the new inserted point by Mes2Complex has a positive weighted distance $\lambda$ from all other existing points. 
Consider a tetrahedron $t$ whose orthocenter is inserted by Mesh3Complex. Since $\rho_{0}>1$, closest point distance cannot decrease when $t$ has all vertices unweighted. When $t$ has a weighted vertex, the inserted orthocenter has a positive weighted distance from all other existing points. This is because the orthocenter is inserted only if it lies at least $2 r$ distance away from any protecting ball of radius $r$. In sum, all points are inserted with a fixed lower bound on their distances to all existing points. A standard packing argument establishes termination of the refinement process.

\section{Topology guarantees}

Let $M$ denote the output mesh of DelPSC. Property T1 follows immediately from Lemma 3.1.

TheOREM 6.1. M satisfies T1.

Theorem 6.2. M satisfies T2.

Proof.

Case(i): $\sigma \in \mathcal{D}_{\leqslant 1}$. It follows trivially from property $\mathrm{T} 1$.

Case(ii): $\sigma \in \mathcal{D}_{2}$. At the end of Mesh2Complex if $\left.\mathrm{Skl}^{2} S\right|_{\sigma}$ is not empty, the Disk_Condition ensures that $\left.\mathrm{Skl}^{2} S\right|_{\sigma}$ is a simplicial complex where each vertex $v$ belongs to $\sigma$ and has a 2-disk as its star. If $\sigma$ has a non-empty boundary, the weighted points on bd $\sigma$ have some edge in their Voronoi cells which intersects $\sigma$ (by Lemma 3.1). If $\sigma$ has empty boundary, the same is guaranteed by the three weighted points which are initially placed on $\sigma$. In both cases $\left.\mathrm{Skl}^{2} S\right|_{\sigma}$ remains non-empty. Insertions in Mesh3Complex does not disturb $\left.\mathrm{Skl}^{2} S\right|_{\mathrm{bd} \sigma^{\prime}}$ for any $\sigma^{\prime} \in \mathcal{D}_{3}$. Therefore, $\left.\mathrm{Skl}^{2} S\right|_{\sigma}$ retains the disk property at each vertex even after Mesh3Complex terminates. It follows from a result in PL topology that $\left.\mathrm{Skl}^{2} S\right|_{\sigma}$ is a 2-manifold when DelPSC terminates.

Now we show that the boundary of $\left.\mathrm{Skl}^{2} S\right|_{\sigma}$ is $\left.\mathrm{Skl}^{1} S\right|_{\mathrm{bd} \sigma}$. Each vertex $\left.v \in \mathrm{Skl}^{2} S\right|_{\sigma}$ is an interior vertex if and only if it belongs to the interior of $\sigma$. Thus, the only vertices which are on the boundary of the manifold are the vertices on $\mathrm{bd} \sigma$. These are the vertices of $\left.\mathrm{Skl}^{1} S\right|_{\mathrm{bd} \sigma}$ by T1. The edges of $\left.\mathrm{bd} \mathrm{Skl}^{2} S\right|_{\sigma}$ can then connect only vertices in $\left.\mathrm{Skl}^{1} S\right|_{\text {bd } \sigma}$. Because of disk condition, these edges cannot connect non adjacent vertices in $\mathrm{bd} \sigma$. Therefore, they connect only adjacent vertices on $\mathrm{bd} \sigma$ and are exactly the edges of $\left.\mathrm{Skl}^{1} S\right|_{\mathrm{bd} \sigma}$ by $\mathrm{T} 1$.

Case (iii): $\sigma \in \mathcal{D}_{3}$. Let $K=\left.\mathrm{Skl}^{3} S\right|_{\sigma}$. First we argue that if $K$ is not empty then it has vertices only in $\sigma$. Suppose not. Then there is a tetrahedron in $K$ one of whose vertex, say $p$, is in $\sigma^{\prime} \in \mathcal{D}$ where $\sigma^{\prime} \nsubseteq \sigma$. The dual Voronoi vertex, say $v$, of this tetrahedron is in $\sigma$ by definition. The Voronoi cell of $p$ intersects both $\sigma$ and $\sigma^{\prime}$. Therefore there is a Voronoi edge of this Voronoi cell that intersects bd $\sigma$. But then $\left.\mathrm{Skl}^{2} S\right|_{\sigma}$ has a triangle with vertex $p \in \sigma^{\prime}$. This contradicts property T2.

Next we establish that $\left.\mathrm{Skl}^{2} S\right|_{\mathrm{bd} \sigma}$ is a 2-manifold which will help proving $K$ is a 3-manifold. By definition $\left.\mathrm{Skl}^{2} S\right|_{\mathrm{bd} \sigma}=\left.\cup \mathrm{Skl}^{2} S\right|_{\sigma_{i}}$ where each $\sigma_{i}$ is a 2-face in bd $\sigma$. By property $\mathrm{T} 2$ any vertex $\left.p \in \mathrm{Skl}^{2} S\right|_{\mathrm{bd} \sigma}$ is in some $\sigma^{\prime} \subseteq \mathrm{bd} \sigma$. If $p$ is in the interior of $\sigma^{\prime} \operatorname{Umb}_{\mathrm{bd} \sigma}(p)$ is a 2-disk by property T2. If $p$ is in bd $\sigma^{\prime}$ we have two cases. If $p$ is not a 0 -face, it is incident to exactly two 2 -faces, $\sigma^{\prime}$ and, say $\sigma^{\prime \prime}$, in $\operatorname{bd} \sigma$. The two 2-disks $\operatorname{Umb}_{\sigma^{\prime}}(p)$ and $\operatorname{Umb}_{\sigma^{\prime \prime}}(p)$ meet along two edges to form a 2-disk with $p$ in its interior. If $p$ is a 0 -face, this construction generalizes to all 2 -faces incident to $p$ on bd $\sigma$. All 2-disks around $p$ meet along common edges to form a 2-disk with $p$ in its interior. Therefore, all vertices in $\left.\mathrm{Skl}^{2} S\right|_{\text {bd } \sigma}$ have a star which is a closed 2-disk with the vertex in its interior. It implies from PL topology that $\left.\mathrm{Skl}^{2} S\right|_{\mathrm{bd} \sigma}$ is a closed 2-manifold. 


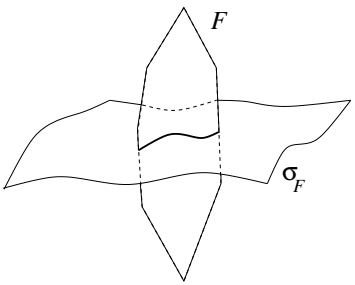

(a)

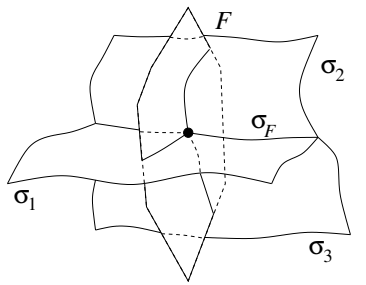

(b)

Figure 4: $F$ is a Voronoi facet $(k=2)$. In (a), $F$ intersects a 2 -face $(j=2)$ in a closed topological interval (1-ball). Voronoi edges $(k=1)$ intersect $\sigma$ in a single point, a 0-ball. In (b), $F$ intersects the 1-face $\left(\sigma_{F}\right)$ in a single point, and for $1 \leqslant i \leqslant 3, F \cap \sigma_{i}$ are closed topological 1-balls. $F$ intersects only 2 -faces that are incident to $\sigma_{F}$.

Now we show that bd $K=\left.\mathrm{Skl}^{2} S\right|_{\mathrm{bd} \sigma}$ as required. Let $T$ be the set of triangles in $K$ that are incident to only one tetrahedron in $K$. We claim that $T=\left.\mathrm{Skl}^{2} S\right|_{\mathrm{bd} \sigma}$. The dual Voronoi edge of any triangle $t \in T$ intersects bd $\sigma$. Otherwise, its two endpoints lie in $\sigma$ whose two dual tetrahedra being incident to $t$ contradict that $t$ is incident only to one tetrahedron. Therefore, $\left.t \in \mathrm{Skl}^{2} S\right|_{\text {bd } \sigma}$ by definition. It follows that $\left.T \subseteq \mathrm{Skl}^{2} S\right|_{\mathrm{bd} \sigma}$. Being on the boundary of a union of tetrahedra, each edge of the triangles in $T$ is incident to positive and even number of triangles in $T$. If $T$ is a strict subset of $\left.\mathrm{Skl}^{2} S\right|_{\mathrm{bd} \sigma}$ some edge in $T$ will have only a single triangle incident to it since $\left.\mathrm{Skl}^{2} S\right|_{\mathrm{bd} \sigma}$ is a connected closed manifold. Therefore $T$ is exactly equal to $\left.\mathrm{Skl}^{2} S\right|_{\mathrm{bd} \sigma}$.

To prove T3 we need a result of Edelsbrunner and Shah [15] about the topological ball property (TBP). For smooth surface meshing, the TBP played a key role in proving topological guarantees $[4,10]$. It turns out that an extended version of the TBP (also given in [15]) is needed to prove topological guarantees for PSCs. It can be shown that the following two properties P1 and P2 imply the extended TBP [8]. Therefore, according to Edelsbrunner-Shah [15] result, Del $\left.S\right|_{\mathcal{D}}$ has underlying space homeomorphic to the $|\mathcal{D}|$ if $\mathrm{P} 1$ and P2 hold (see Figure 4). Let $F$ be a $k$-face of Vor $S$ where $S$ is the output vertex set.

(P1) If $F$ intersects an element $\sigma \in \mathcal{D}_{j} \subseteq \mathcal{D}$, the intersection is a closed $(k+j-3)$-ball.

(P2) There is a unique lowest dimensional element $\sigma_{F} \in \mathcal{D}$ so that $F$ intersects $\sigma_{F}$ and only elements that are incident to $\sigma_{F}$.

Lemma 3.1 and Lemma 5.2 together almost provide condition P1 except that the precondition of intersecting Voronoi edges of Lemma 5.2 needs to be proved and the case of a surface patch avoiding all Voronoi edges as in Figure 3(right) needs to be avoided. These two facts are proved in Lemma 6.1 and Lemma 6.2 respectively. Notice that we require stronger condition on triangle sizes for proving these results. Both Lemma 6.1 and Lemma 6.2 require that all restricted triangles have small sizes in contrast to restricted triangles incident to a particular point as in Lemma 5.2. It is interesting to note that the termination guarantee does not require this stronger condition.

Lemma 6.1. There exists a constant $\lambda>0$ for which following holds. If size $\left(t, \sigma^{\prime}\right)<\lambda$ for each tuple $\left(t, \sigma^{\prime}\right),\left.t \in \mathrm{Skl}^{2} S\right|_{\sigma^{\prime}}$, then for each point $p \in S$ and each 2 -face $\sigma$ where $p \in \sigma$, an edge of $V_{p}$ intersects $\sigma$.

Proof. Suppose that no edge of $V_{p}$ intersects $\sigma$. Let $q \in \sigma$ be a weighted point. By the initialization step each 2-face has at least one such point. Because of Lemma 3.1 an edge of $V_{q}$ has to intersect $\sigma$. 
Consider walking on a path in $\sigma$ from $p$ to $q$. Let $p=p_{0}, p_{1}, . ., p_{k}=q$ be sequence of vertices whose Voronoi cells are encountered along this walk. Since no edge of $V_{p}$ intersects $\sigma$ and some edge of $V_{q}$ intersects $\sigma$, there exists two consecutive vertices $p_{i}$ and $p_{i+1}$ in this sequence so that no edge of $V_{p_{i}}$ intersects $\sigma$ whereas some edge of $V_{p_{i+1}}$ does intersect $\sigma$. By Lemma 5.2 we can claim that $\sigma_{p_{i+1}}$ is a disk. A boundary cycle of $\sigma_{p_{i}}$ overlaps with the boundary of $\sigma_{p_{i+1}}$. This is impossible as the curves on the boundary of $\sigma_{p_{i+1}}$ intersect Voronoi edges whereas those on the boundary of $\sigma_{p_{i}}$ do not.

Lemma 6.2. There exists a constant $\lambda>0$ such that if $\operatorname{size}\left(t, \sigma^{\prime}\right)<\lambda$ for each tuple $\left(t, \sigma^{\prime}\right),\left.t \in \mathrm{Skl}^{2} S\right|_{\sigma^{\prime}}$, then for each point $p$ and 2 -face $\sigma$ where $p \in \sigma$

(i) $\left.V_{p}\right|_{\sigma}$ is a 2-disk;

(ii) any edge of $V_{p}$ intersects $\left.V_{p}\right|_{\sigma}$ at most once;

(iii) any facet of $V_{p}$ intersects $\left.V_{p}\right|_{\sigma}$ in an empty set or an open curve.

(iv) $V_{p}$ does not intersect any $\sigma^{\prime}$ where $p \notin \sigma^{\prime}$.

Proof. Recall that $\sigma_{p}$ denotes the connected component in $\left.V_{p}\right|_{\sigma}$ containing $p$. Because of Lemma 6.1 we can apply Lemma 5.2 to claim that $\sigma_{p}$ satisfies properties (i)-(iii) for each $\sigma$ containing $p$. Therefore, it suffices to prove that $\left.V_{p}\right|_{\mathcal{D}}=\bigcup_{\sigma \ni p} \sigma_{p}$ to claim (i)-(iv).

Assume to the contrary that $\left.\bigcup \sigma_{p} \subset V_{p}\right|_{\mathcal{D}}$. By Lemma 5.2, $\sigma_{p}=V_{p} \cap B \cap \sigma$ and no edge of $V_{p}$ intersects $\mathcal{D}$ outside $B$. This implies that for any connected component $\left.C \in V_{p}\right|_{\mathcal{D}} \backslash \bigcup \sigma_{p}$, bd $C$ lies inside facets of $V_{p}$. Also, $V_{p}$ can intersect a 1-face only if it contains $p$ (by Lemma 3.1). Therefore, bd $C$ cannot have endpoints implying that bd $C$ is a set of closed curves not intersecting any Voronoi edge.

Let $C \subset \sigma^{\prime}$. Voronoi cells partition $\sigma^{\prime}$. A path on $\sigma^{\prime}$ from bd $C$ to a sample point $q \in \sigma^{\prime}$ passes through the connected components of this partition. We must encounter two adjacent components along this path, say $C^{\prime}$ and $C^{\prime \prime}$, where $C^{\prime} \neq \sigma_{s}^{\prime}$ for any $s$ and $C^{\prime \prime}=\sigma_{r}^{\prime}$ for some $r \in \sigma^{\prime}$. This is because the first and last components satisfy this property. Then, we reach a contradiction since bd $C^{\prime \prime}=\mathrm{bd} \sigma_{r}^{\prime}$ intersects Voronoi edges by Lemma 5.2 whereas bd $C^{\prime}$ does not.

Lemma 3.1 and Lemma 6.2 establish two facts for $\sigma \in \mathcal{D}_{\leqslant 2}$ before Mesh3Complex begins : (i) $\sigma$ intersects $V_{p}$ if and only if $p \in \sigma$, and (ii) property $\mathrm{P} 1$ holds for $\sigma$. Since Mesh3Complex does not insert any point which encroaches a triangle in $\left.\mathrm{Skl}^{2} S\right|_{\sigma}$, (i) and (ii) still hold for any $\sigma \in \mathcal{D}_{\leqslant 2}$. This means (i) also holds for any 3-face $\sigma$ at termination. Let $p$ be in the interior of $\sigma \in \mathcal{D}_{3}$. Then, $V_{p}$ cannot intersect $\operatorname{bd} \sigma$ due to (i). In this case $\sigma$ satisfies $\mathrm{P} 1$ with $V_{p}$ trivially. If $p$ is in bd $\sigma$, the intersection $V_{p} \cap \sigma$ should satisfy P1 again since bd $\sigma \cap V_{p}$ does so. This establishes P1. Next lemma establishes Property P2.

LEMma 6.3. There exists a $\lambda>0$ so that if $\operatorname{size}(t, \sigma)<\lambda$ for each tuple $(t, \sigma),\left.t \in \mathrm{Skl}^{2} S\right|_{\sigma}$, then the following holds. Let $F$ be a $k$-face in Vor $S$. There is an element $\sigma_{F} \in \mathcal{D}$ so that $F$ intersects $\sigma_{F}$ and only elements in $\mathcal{D}$ that have $\sigma_{F}$ on their boundary.

Proof. Case 1: $F$ is a Voronoi cell $V_{p}$. Let $\sigma_{F} \in \mathcal{D}$ be the lowest dimensional element containing $p$. We claim all elements in $\mathcal{D}$ intersecting $F$ have $\sigma_{F}$ in their boundaries and thus $\sigma_{F}$ is unique. If not, let there be another $\sigma^{\prime} \in \mathcal{D}$ where $\sigma_{F} \not \subset$ bd $\sigma^{\prime}$. Notice that $p \notin \sigma_{F} \cap \sigma^{\prime}$ since otherwise $\sigma_{F} \cap \sigma^{\prime}$ is either an element of $\mathcal{D}$ whose dimension is lower than $\sigma_{F}$ or $\sigma_{F} \cap \sigma^{\prime}=\sigma_{F}$ both of which are impossible. It follows that $p \notin \sigma^{\prime}$. But we already argued above that $V_{p}$ intersects only elements in $\mathcal{D}$ that contain $p$.

Case 2: $F$ is a Voronoi facet $V_{p q}$. Let $\sigma_{F}$ be a lowest dimensional element that $F$ intersects. Assume there is another $\sigma^{\prime}$ intersecting $F$ where $\sigma_{F} \not \subset$ bd $\sigma^{\prime}$. We go over different dimensions of $\sigma^{\prime}$ 
each time reaching a contradiction. If $\sigma^{\prime} \in \mathcal{D}_{3}$, bd $\sigma^{\prime}$ must intersect $F$. Otherwise, $F$ intersects only $\sigma^{\prime}$ contradicting that it also intersects $\sigma_{F} \neq \sigma^{\prime}$. Renaming bd $\sigma^{\prime}$ as $\sigma^{\prime}$ we can use the contradiction reached for the case below.

Assume $\sigma^{\prime} \in \mathcal{D}_{\leqslant 2} ; \sigma^{\prime}$ intersects $F$ and does not contain $\sigma_{F}$ on its boundary. Two cases can arise. Either (i) $\sigma_{F}$ and $\sigma^{\prime}$ are disjoint within $V_{p}$ or $V_{q}$, or (ii) $\sigma_{F}$ and $\sigma^{\prime}$ have a common boundary in $V_{p}$ and $V_{q}$. Case (i) cannot happen due to the claim in Case 1. For (ii) to happen both $p$ and $q$ have to be on the common boundaries of $\sigma_{F}$ and $\sigma^{\prime}$. This means that $p$ and $q$ have to be on some element in $\mathcal{D}_{\leqslant 1}$. Observe that $p$ and $q$ are non-adjacent since otherwise $V_{p q}$ has to intersect the common boundary of $\sigma_{F}$ and $\sigma^{\prime}$ whose dimension is lower than that of $\sigma_{F}$. But this would contradict the disk condition that no two non-adjacent vertices in $\mathcal{D}_{1}$ is connected by a restricted edge.

The above argument implies that all elements intersecting $F$ have $\sigma_{F}$ as a subset.

Case 3: $F$ is a Voronoi edge. Certainly $F$ cannot intersect a 2-face $\sigma_{F}$ more than once due to Lemma 6.2. The other possibility is that $F=V_{t},\left.t \in \mathrm{Skl}^{2} S\right|_{\sigma_{F}}$, and $F$ intersects $\sigma^{\prime} \neq \sigma_{F}$. But then a Voronoi cell adjacent to $F$ would intersect two 2 -faces $\sigma_{F}$ and $\sigma^{\prime}$ and $t$ is in both $\left.\mathrm{Skl}^{2} S\right|_{\sigma_{F}}$ and $\left.\mathrm{Skl}^{2} S\right|_{\sigma^{\prime}}$ violating the disk condition.

\section{TheOREM 6.3. M satisfies T3.}

Proof. For sufficiently small $\lambda>0$, the triangles in $\left.\mathrm{Skl}^{2} S\right|_{\mathcal{D}}$ satisfy the conditions for Lemma 6.2 and Lemma 6.3. This means that properties P1 and P2 are satisfied when $\lambda$ is sufficiently small. Also when $\mathrm{P} 1$ and $\mathrm{P} 2$ are satisfied $\left.\bigcup_{i} \mathrm{Skl}^{i} S\right|_{\mathcal{D}}=\left.\operatorname{Del} S\right|_{\mathcal{D}}$. It follows that Edelsbrunner-Shah conditions are satisfied for the output $M$ of DelPSC. Thus, $M$ has an underlying space homeomorphic to $|\mathcal{D}|$. The homeomorphism constructed by Edelsbrunner and Shah actually respects the stratification, that is, for each $\sigma \in \mathcal{D}_{i},\left.\mathrm{Skl}^{i} S\right|_{\sigma}$ is homeomorphic to $\sigma$. Furthermore, property T2 holds for any output of DelPSC. This means, for $0 \leqslant i \leqslant 2,\left.\mathrm{Skl}^{i} S\right|_{\sigma}=\left.\mathrm{Skl}^{i-1} S\right|_{\mathrm{bd} \sigma}$ and $\left.\mathrm{Skl}^{i} S\right|_{\sigma}$ has vertices only in $\sigma$. We can claim the same condition even for $i=3$ since $\left.\mathrm{Skl}^{3} S\right|_{\sigma}, \sigma \in \mathcal{D}_{3}$, is not empty at the end of Mesh2Complex when $\lambda$ is sufficiently small. A Voronoi edge intersecting bd $\sigma$ cannot intersect it more than once as we argued in Case 3 of Lemma 6.3.

\section{Conclusions}

We have presented a practical algorithm to mesh a wide variety of geometric domains with Delaunay refinement technique. The output mesh maintains a manifold property and with increasing level of refinement captures the topology of the input. An interesting aspect of the algorithm is that the input 'non-smooth features' are preserved in the output.

A number of experimental results that validate our claims. This is the first practical algorithm to produce Delaunay meshes for a large class of three dimensional geometric domains with theoretical guarantees. It can handle arbitrarily small input angles and preserve input features. When applied to volumes, the algorithm guarantees bounded radius-edge ratio for most of the tetrahedra except near boundary. It can be easily extended to guarantee bounded aspect ratio for most triangles and bounded radius-edge ratio for most tetrahedra except the ones near non-smooth elements.

An obvious open question is to analyze the time and space complexity of the algorithm. Is it possible to claim non-trivial or even optimal bounds for these complexities. Very few results exist for optimal Delaunay refinement [16]. Finally, can the algorithm be improved even more by incorporating easier primitives? 


\section{References}

[1] N. Amenta and M. Bern. Surface reconstruction by Voronoi filtering. Discr. Comput. Geom. 22 (1999), 481-504.

[2] N. Amenta, S. Choi, T. K. Dey and N. Leekha. A simple algorithm for homeomorphic surface reconstruction. Internat. J. Comput. Geom. Applications 12 (2002), 125-141.

[3] J.-D. Boissonnat and S. Oudot. Provably good sampling and meshing of Lipschitz surfaces. Proc. 22nd Ann. Sympos. Comput. Geom. (2006), 337-346.

[4] J.-D. Boissonnat and S. Oudot. Provably good surface sampling and meshing of surfaces. Graphical Models 67 (2005), 405-451.

[5] H.-L. Cheng, T. K. Dey, H. Edelsbrunner, and J. Sullivan. Dynamic skin triangulation. Discrete Comput. Geom. 25 (2001), 525-568.

[6] S.-W. Cheng, T. K. Dey, and J. A. Levine. A practical Delaunay meshing algorithm for a large class of domains. Proc. 16th Internat. Meshing Roundtable (2007), to appear.

[7] S.-W. Cheng and S.-H. Poon. Three-dimensional Delaunay mesh generation. Discrete Comput. Geom. 36 (2006), 419-456.

[8] S.-W. Cheng, T. K. Dey, and E. A. Ramos. Delaunay refinement for piecewise smooth complexes. Proc. 18th Ann. ACM-SIAM Sympos. Discrete Algorithms (2007), 1096-1105.

[9] S.-W. Cheng, T. K. Dey, E. A. Ramos and T. Ray. Quality meshing for polyhedra with small angles. Internat. J. Comput. Geom. Appl. 15 (2005), 421-461.

[10] S.-W. Cheng, T. K. Dey, E. A. Ramos and T. Ray. Sampling and meshing a surface with guaranteed topology and geometry. Proc. 20th Ann. Sympos. Comput. Geom. (2004), 280-289.

[11] L. P. Chew. Guaranteed-quality triangular meshes. Report TR-98-983, Comput. Sci. Dept., Cornell Univ., Ithaca, New York, 1989.

[12] L. P. Chew. Guaranteed-quality mesh generation for curved surfaces. Proc. 9th Ann. Sympos. Comput. Geom. (1993), 274-280.

[13] T. K. Dey, C. Bajaj and K. Sugihara. On good triangulations in three dimensions. Internat. J. Comput. Geom. 2 (1992), 75-95.

[14] T. K. Dey, G. Li, and T. Ray. Polygonal surface remeshing with Delaunay refinement. Proc. 14th Internat. Meshing Roundtable (2005), 343-361.

[15] H. Edelsbrunner and N. Shah. Triangulating topological spaces. Internat. J. Comput. Geom. Appl. 7 (1997), 365-378.

[16] S. Har-Peled and A. Üngör. A time optimal Delaunay refinement algorithm in two dimensions. Proc. Ann. Sympos. Comput. Geom. (2005), 228-236.

[17] X.-Y. Li and S.-H. Teng. Generating well-shaped Delaunay meshes in 3D. Proc. 12th. Ann. ACM-SIAM Sympos. Discrete Algorithm (2001), 28-37.

[18] G. L. Miller, D. Talmor, S.-H. Teng and N. Walkington. A Delaunay based numerical method for three dimensions: generation, formulation, and partition. Proc. 27th Ann. ACM Sympos. Theory Comput. (1995), 683-692.

[19] S. Oudot, L. Rineau, and M. Yvinec. Meshing volumes bounded by smooth surfaces. Proc. 14th Internat. Meshing Roundtable (2005), 203-219.

[20] S. Pav and N. Walkington. Robust three dimensional Delaunay refinement. 13th Internat. Meshing Roundtable 2004.

[21] J. Ruppert. A Delaunay refinement algorithm for quality 2-dimensional mesh generation. J. Algorithms, 18 (1995), 548-585.

[22] J. R. Shewchuk. Tetrahedral mesh generation by Delaunay refinement. Proc. 14th Ann. Sympos. Comput. Geom. (1998), 86-95.

[23] J. R. Shewchuk. Mesh generation for domains with small angles. Proc. 16th Ann. Sympos. Comput. Geom. (2000), 1-10. 